\title{
Australian pharmacists: ready for increased non-prescription medicines reclassification
}

Denise L. Hope, ${ }^{a}$ Phillip Woods, ${ }^{b}$ Amary Mey, ${ }^{c}$ Fiona S. Kelly, ${ }^{d}$ James Townshend,,${ }^{e}$ Lyndsee M. Baumann-Birkbeck, ${ }^{f}$ Michelle A. King ${ }^{g}$

a,c,f,g School of Pharmacy and Pharmacology, Quality Use of Medicines Network, and Menzies Health Institute Queensland, Griffith University, Queensland 4222 Australia b,d,e School of Pharmacy and Pharmacology, and Quality Use of Medicines Network, Griffith University, Queensland 4222 Australia

ad.hope@griffith.edu.au

bphillip.woods@griffith.edu.au

ca.mey@griffith.edu.au

d.kelly@griffith.edu.au

j.townshend@griffith.edu.au

flyndsee.baumann-birkbeck@griffithuni.edu.au

gmichelle.a.king@griffith.edu.au https://orcid.org/0000-0003-3733-8366

https://orcid.org/0000-0002-0744-1891

https://orcid.org/0000-0002-5339-5101

https://orcid.org/0000-0001-7360-4655

https://orcid.org/0000-0001-5604-7463

https://orcid.org/0000-0001-6890-7838

https://orcid.org/0000-0001-5922-8032

\section{Corresponding author:}

Ms. Denise Hope

School of Pharmacy and Pharmacology, Clinical Sciences 2, G16_3.26, Griffith University, Queensland 4222, Australia

Telephone: $\quad+61755527339$

Facsimile: $\quad+61755529755$

Email: $\quad$ d.hope@griffith.edu.au

All authors devised the study and its methodology, created the questionnaire, sought ethics approval, recruited participants and collected questionnaires electronically. $\mathrm{MK}$ and $\mathrm{DH}$ performed statistical analyses. All authors had access to the data, contributed to all drafts of the paper and approved the final copy for publication. 


\section{Australian pharmacists: ready for increased non-prescription medicines reclassification}

\section{Abstract}

Objectives Reclassification of medicines from prescription to non-prescription increases timely access to treatment, promotes self-management of minor ailments and relieves healthcare system burden. Previous research identified that Australia lagged behind the United Kingdom and New Zealand in medicines reclassification. This study aimed to identify Australian pharmacists' opinions on the current state of medicines reclassification; the prescription medicines consumers requested without prescription; the medicines pharmacists believed should and should not be considered for reclassification; and perceived barriers to reclassification.

Methods A 2016 national online survey that sought pharmacists' opinions on the state of reclassification, perceived barriers to reclassification and readiness of the profession for further reclassification. Pharmacists' comments were invited through open-ended questions.

Key findings Two hundred and thirty-five valid surveys were completed. Respondents practised in community, hospital, consultant and academic contexts, and the majority were female $(58.7 \%, \mathrm{n}=138)$. More than two thirds $(70.66 \%, \mathrm{n}=166)$ of pharmacists reported receiving daily or weekly requests for non-prescription access to prescription medicines. The majority of pharmacists (71.7\%) agreed that the Australian pharmacy profession is ready for further medicines reclassification, guided by patient safety, harm minimisation and medication continuance. The most prominent barrier to further reclassification was opposition from other healthcare professionals.

Conclusions Australian pharmacists believe that their profession has the capacity to safely and effectively manage a wider range of non-prescription medicines through increased reclassification in the contexts of patient safety and risk mitigation. This study has contributed 
to the global conversation on non-prescription medicines access, providing momentum for practice and policy change.

Key Words Australia; pharmacists; health policy; legislation, drug; non-prescription drugs. 


\section{Introduction}

Community access to medicines without prescription can increase timely initiation of effective treatment, promote self-management of minor ailments, and encourage patient autonomy in health decision-making. ${ }^{1,2}$ Responsible self-medication has also been shown to provide significant economic benefits, including improved productivity and cost savings. ${ }^{2-4}$

In Australia and countries with similar health systems, access to medicines is regulated through a classification system or scheduling framework, guided by a medicine's therapeutic purpose, potential for abuse, safety, toxicity and the need for access. ${ }^{5}$ In Australia, unscheduled medicines are considered low risk and are available for general sale from retail outlets, while scheduled medicines are classified into progressively more restrictive schedules under the federal Standard for Uniform Scheduling of Medicines and Poisons (SUSMP). Schedule 2 (S2) Pharmacy Medicines are available only from pharmacies and may be obtained without further necessary intervention; Schedule 3 (S3) Pharmacist Only Medicines are available only from pharmacies and require pharmacist involvement to determine appropriateness and advice to ensure proper use; Schedule 4 (S4) Prescription Only Medicines and Schedule 8 (S8) Controlled Drugs are available only on prescription. S8 medicines have the greatest degree of restriction to reduce potential abuse, misuse and dependence. ${ }^{5}$

An Australian study that reviewed medication access guided by the classification processes in six countries, i.e. Australia, New Zealand, Canada, France and the United Kingdom (UK), reported that the presence of pharmacy-specific schedules facilitated reclassification of medicines into less restrictive schedules, thereby broadening consumer access. ${ }^{6}$ This contrasts with the United States of America (USA) where pharmacy-only schedules do not exist. ${ }^{2}$ A range of other factors have been identified as either enablers or barriers to medicines reclassification. ${ }^{7,8}$ Gauld et al showed that enablers included stakeholders' confidence in regulators and regulator support. ${ }^{7}$ Barriers to reclassification included risk averseness, limited 
trust by regulators in consumers and pharmacists, and 'patch protection' by other healthcare professionals, for example the Australian Medical Association has opposed reclassifications. ${ }^{8}$ These articles identified that Australia had more barriers than enablers when compared with the $\mathrm{UK}^{7}$ and New Zealand. ${ }^{8}$

Notwithstanding these significant barriers, Australian examples of medicines that have been reclassified from S4 to S3 include levonorgestrel for emergency contraception in $2004,{ }^{9}$ proton pump inhibitors for reflux from $2008,{ }^{10}$ ophthalmic chloramphenicol for bacterial conjunctivitis in $2010,{ }^{11}$ oral famciclovir for cold sores in $2012,{ }^{12}$ naloxone for opioid overdose in $2016^{13}$ and ulipristal for emergency contraception in $2017 .{ }^{14}$

Medicines reclassification has also occurred in the direction of increased restriction, primarily due to concerns over medicines abuse, misuse or safety. For example, Australia reclassified flunitrazepam in 1997 and alprazolam in 2014, from S4 to S8, ${ }^{15}$ and codeinecontaining analgesics from S3 to S4 in $2018 .{ }^{16}$ Additionally, Australia reduced pack sizes and reclassified non-prescription pseudoephedrine to $\mathrm{S} 3$ in $2006 .{ }^{16}$ In New Zealand, pseudoephedrine was up-scheduled to prescription only in $2011,{ }^{17,18}$ and in the UK, oral diclofenac was reclassified from non-prescription to prescription-only in 2015 , due to concerns over perceived cardiovascular risk. ${ }^{19}$

When compared with other countries with similar health systems e.g. the UK and New Zealand, Australia's reclassification appears to have fallen behind. ${ }^{8,20,21,22}$ Despite the goal of regulatory harmonisation between Australia and New Zealand, ${ }^{23,24}$ between 2003 and 2013, New Zealand allowed more drugs to be rescheduled than Australia, ${ }^{21}$ including the provision of pharmacist only supply of sildenafil for erectile dysfunction, triptans for migraine, trimethoprim for urinary tract infection (UTI) ${ }^{25}$ and oseltamivir for influenza. ${ }^{8,17,20,26}$ These medicines are currently S4 in Australia, suggesting that consumers may face unnecessary barriers to timely access for some medicines. 
There are additional medicines that have been reclassified in other countries that remain prescription only in Australia. ${ }^{22,27,28}$ For example, New Zealand has recently approved selected oral contraceptives for non-prescription access from specially trained pharmacists ${ }^{29}$ while Australia rejected applications for reclassification of oral contraceptives and has only allowed pharmacist provision of certain oral contraceptives under comparatively strict conditions of the Continued Dispensing Initiative. ${ }^{30}$ Continued Dispensing occurs within the Pharmaceutical Benefits Scheme (PBS) and allows pharmacists to provide medication continuance of subsidised oral contraceptives and 'statins', without prescription once every 12 months if patients meet particular criteria. ${ }^{30}$ This approach is similar to prescribing models such as the Chronic Medication Service in Scotland..$^{31}$

Recent research to examine New Zealand pharmacists' views on reclassification of certain medicines indicated both motivation and readiness. ${ }^{32}$ The extent of Australian pharmacists' perceived readiness for a greater role in providing increased consumer access to medicines that are presently prescription only is under explored. Pilot research indicated that pharmacists often received requests for non-prescription access to prescription medicines and supported reclassification, underpinned by motivation to facilitate consumers' self-management and medication adherence. ${ }^{33}$ However, the small study sample involved only community pharmacy staff and may not be generalisable to all practice contexts. It is timely to more broadly explore Australian pharmacists' perspectives, particularly given the February 2019 introduction of Appendix M to the SUSMP by the Australian Therapeutics Goods Administration. Appendix $\mathrm{M}$ has been proposed as a mechanism to allow provision of selected S4 medicines by a pharmacist without prescription and will list S3 medicines with additional controls or supply requirements. ${ }^{34}$

The aim of this study was to explore and identify Australian pharmacists' opinions on the current state of medicines reclassification; the type of prescription medicines that consumers 
request to access without prescription; the frequency of requests; the medicines that pharmacists think should and should not be considered for reclassification; the perceived barriers to reclassification; and the readiness of the profession for future reclassification of prescription to non-prescription medicines in Australia. The intended outcome was to inform the national and global conversations regarding consumer access to medicines.

\section{Methods}

A survey was developed, informed by the literature and pilot qualitative research. ${ }^{33}$ The survey comprised three sections. Section 1 gathered demographic information (gender, length of practice, practice location, role and practice context), sought opinions on Australia's current state a medicines reclassification using 5 -point Likert scale responses $(1=$ strongly disagree and 5 = strongly agree) and asked how often participants were asked for non-prescription access to prescription medicines (daily, weekly, monthly, every few months, rarely or never). Section 1 also invited free-text responses for the most common medicines asked for, medicines the participant thought should be made available without prescription, and why. Section 2 sought opinions related to 17 different medicines/medicine classes that were available without prescription in countries with similar health systems to Australia. Data generated from Section 2 have been reported elsewhere ${ }^{35}$ and will not be reported in this manuscript. Section 3 sought opinions on the readiness of the Australian pharmacy profession for reclassification, using a 10 -point scale $(1=$ not at all ready and $10=$ completely ready $)$ used for assessing motivational readiness for change. ${ }^{36}$ Section 3 also sought opinions on perceived barriers to change with a 5-point Likert scale and invited free-text responses on the classes of prescription medicines participants thought should never be considered for reclassification and why. The survey was piloted with pharmacists from an academic and/or community pharmacy background and items were reviewed for face and content validity. 
The online JotForm survey was administered nationally between August and December 2016. The survey was promoted by a national pharmacy organisation via a continuing professional e-learning website. Strategies used to enhance response rates for online surveys included selection of a topical survey subject, official sponsorship via pharmacy industry channels, use of multiple online channels for recruitment, limiting the length of the survey and a prize draw to incentivise participation. ${ }^{37}$ Institutional ethical clearance was obtained (PHM/04/15/HREC).

Data were exported to Microsoft Excel. Responses from non-pharmacist pharmacy staff ( $n=12,4.9 \%$ of 247 responses) were excluded from data analysis as being poorly representative. Responses to free-text questions were coded thematically by two researchers and confirmed by a third. Pharmacists identified individual medicines or medicine classes as medicines that should or should not be reclassified and these were coded according to the Anatomical Therapeutic Chemical classification system hierarchy, ${ }^{38}$ using the process outlined above. SPSS 22 was used to calculate means and medians of Likert scale responses, frequencies and percentages. Likert scale responses were either reported as aggregated into agree/strongly agree or neither agree or disagree, or disagree/strongly disagree, or as means and medians to provide an overview of level of agreement with particular statements.

\section{Results}

Results included 235 valid survey responses from pharmacists, working in community, hospital, consultant and academic practice contexts. Participants were predominantly female (Table 1). All Australian states and territories were represented. 


\begin{tabular}{|c|c|c|c|}
\hline $\begin{array}{l}\text { Demographic } \\
\text { Characteristic }\end{array}$ & & $\mathbf{n}$ & $(\%)$ \\
\hline \multicolumn{4}{|l|}{ Gender $(n=235)$} \\
\hline & Male & 97 & $(41.3)$ \\
\hline & Female & 138 & $(58.7)$ \\
\hline \multicolumn{4}{|c|}{ Length of Practice $(n=235)$} \\
\hline & Less than 5 years & 21 & $(8.9)$ \\
\hline & $6-10$ years & 62 & $(26.4)$ \\
\hline & 11-20 years & 51 & $(21.7)$ \\
\hline & 21-30 years & 30 & $(12.8)$ \\
\hline & $31-40$ years & 53 & $(22.6)$ \\
\hline & $41-50$ years & 8 & $(3.4)$ \\
\hline & More than 51 years & 10 & $(4.3)$ \\
\hline \multicolumn{4}{|c|}{ State or Territory $(n=235)$} \\
\hline & Australian Capital Territory & 13 & $(4.5)$ \\
\hline & New South Wales & 97 & $(33.8)$ \\
\hline & Northern Territory & 4 & $(1.4)$ \\
\hline & Queensland & 96 & $(33.4)$ \\
\hline & South Australia & 10 & $(3.5)$ \\
\hline & Tasmania & 7 & $(2.4)$ \\
\hline & Victoria & 40 & $(13.9)$ \\
\hline & Western Australia & 19 & $(6.6)$ \\
\hline \multicolumn{4}{|c|}{ Practice Role $(n=231)$} \\
\hline & Student Pharmacist & 2 & $(0.9)$ \\
\hline & Intern Pharmacist & 8 & $(3.4)$ \\
\hline & Dispensing Pharmacist & 80 & $(34.0)$ \\
\hline & Forward Pharmacist & 96 & $(40.9)$ \\
\hline & Consultant Pharmacist & 16 & $(6.8)$ \\
\hline & Other (including academia) & 29 & $(12.3)$ \\
\hline
\end{tabular}




\section{Current state of medicines classification}

Opinions on whether 'the current rate of down-scheduling limited consumers' access to medicines' ( $n=228)$ were polarised, with 95 participants $(41.7 \%)$ indicating that they disagree or strongly disagree with the statement while $88(38.6 \%)$ indicated that they agreed or strongly agreed with the statement. (NB the term down-scheduling indicates reclassification to less restrictive medicines access. It is commonly used by Australian health practitioners and can be used interchangeably with reclassification, providing directional context to reclassification.) Responses to the statement depicting the current scheduling as providing appropriate access to medicines $(\mathrm{n}=226)$ were also polarised, with 74 participants $(32.7 \%)$ disagreeing or strongly disagreeing while 105 (46.5\%) agreed or strongly agreed.

\section{Requests for non-prescription supply of Prescription Only Medicines}

Respondents indicated that they were often asked for non-prescription access to a wide range of prescription medicines, with $70.6 \%(\mathrm{n}=166)$ indicating that they had been asked at least once weekly. The most commonly requested medicines were anti-infectives $(25.8 \%$, $\mathrm{n}=161$, combining antibiotics, antivirals and antifungals), analgesics, oral contraceptives, corticosteroids (mainly topical and inhaled) and anti-emetics. Reported requests included both increased quantities and/or higher strengths of existing non-prescription analgesics, or access to prescription only analgesics, such as tramadol and celecoxib. Response to the statement 'Australia is aligned with down-scheduling in countries with similar health systems' $(n=231)$, indicated many were neutral $(\mathrm{n}=84,36.4 \%)$.

\section{Medicines that should be down-scheduled}

The majority of respondents $(\mathrm{n}=176,74.9 \%)$ identified medicines that they considered should be down-scheduled. The remaining respondents considered reclassification unnecessary 
$(n=36,15.3 \%)$, were uncertain $(n=4,1.7 \%)$ or did not answer $(n=19,8.1 \%)$. Table 2 summarises the medicines most frequently identified as potential candidates for downscheduling and exemplar comments that justified these choices.

Table 2: Most Frequently Reported Prescription Medicine Classes for Down-Scheduling

\begin{tabular}{lrc}
\hline Classification & n & $(\mathbf{( \% )}$ \\
\hline Anti-infectives & 112 & $(25.9)$ \\
Contraceptives & 73 & $(16.9)$ \\
Corticosteroids & 67 & $(15.5)$ \\
Lipid lowering agents & 31 & $(7.2)$ \\
Antiemetics & 29 & $(6.7)$ \\
Analgesics & 21 & $(4.9)$ \\
Antihypertensives & 19 & $(4.4)$ \\
Erectile dysfunction drugs & 13 & $(3.0)$ \\
Proton pump inhibitors & 13 & $(3.0)$ \\
Antimigraine drugs & 8 & $(1.9)$ \\
Vaccines & 6 & $(1.4)$ \\
Other & 40 & $(9.3)$ \\
$*$ A total of 432 medicines were reported & & \\
\hline
\end{tabular}

Exemplar Respondent Quotes Supporting Opinions for Down-Scheduling

Medication safety:

If the medication is safe; and treatment without regular review by a health professional is safe, then a change in schedule should be considered on its merits. (ID 259)

Medicines continuance:

Up to weeks supply of most medication required for continued treatment in emergency when contact with prescriber is not possible. This would relieve pressure on emergency departments. (ID 272)

Antibiotics were the most frequently proposed drug class, spanning topical, otic and selected systemic medicines. Justifications for potential down-scheduling included improved consumer access to medicines; the promotion of safe access to effective medicines for acute conditions; alignment with other countries; and consumer and healthcare cost savings. Some supported the concept of medication continuance without a new prescription for established medication regimens. 
Only a small number of participants $(3.0 \%, \mathrm{n}=13)$ identified erectile dysfunction drugs as having been requested by consumers and as potential candidates for reclassification, whereas tamsulosin for the urinary symptoms of benign prostatic hyperplasia was not identified. Respondents prioritised medicines that would facilitate medication continuance for ongoing chronic or stabilised conditions, such as asthma, e.g. inhaled corticosteroids, or hypertension, e.g. antihypertensives. Continuity of patient care was often discussed in qualifying text responses to support candidates for potential reclassification.

\section{Medicines that should never be down-scheduled}

Most respondents $(n=207,88.1 \%)$ provided detailed suggestions on medicines that should not be considered for reclassification and these reflected a wide range of drugs and drug classes ( $\mathrm{n}=577$ classifiable medicines), with one respondent listing 15 different classes of medicines.

Table 3 summarises the medicines most frequently identified as never to be down-scheduled and associated exemplar comments. 
Table 3: Most Frequently Reported Prescription Medicine Classes Not for Down-Scheduling

\section{Classification}

Psychotropic drugs

Cardiovascular drugs

Anti-infectives

Analgesics

Antineoplastics and immunomodulators

Neurologicals

Antidiabetic drugs

Endocrine drugs

Other n $(\%) *$

$153 \quad(26.5)$

$105(18.2)$

$86 \quad(14.9)$

$85 \quad(14.7)$

$53 \quad(9.2)$

$36 \quad(6.2)$

$24 \quad(4.2)$

17 (2.9)

$18 \quad(3.1)$

*A total of 577 classifiable medicines were reported

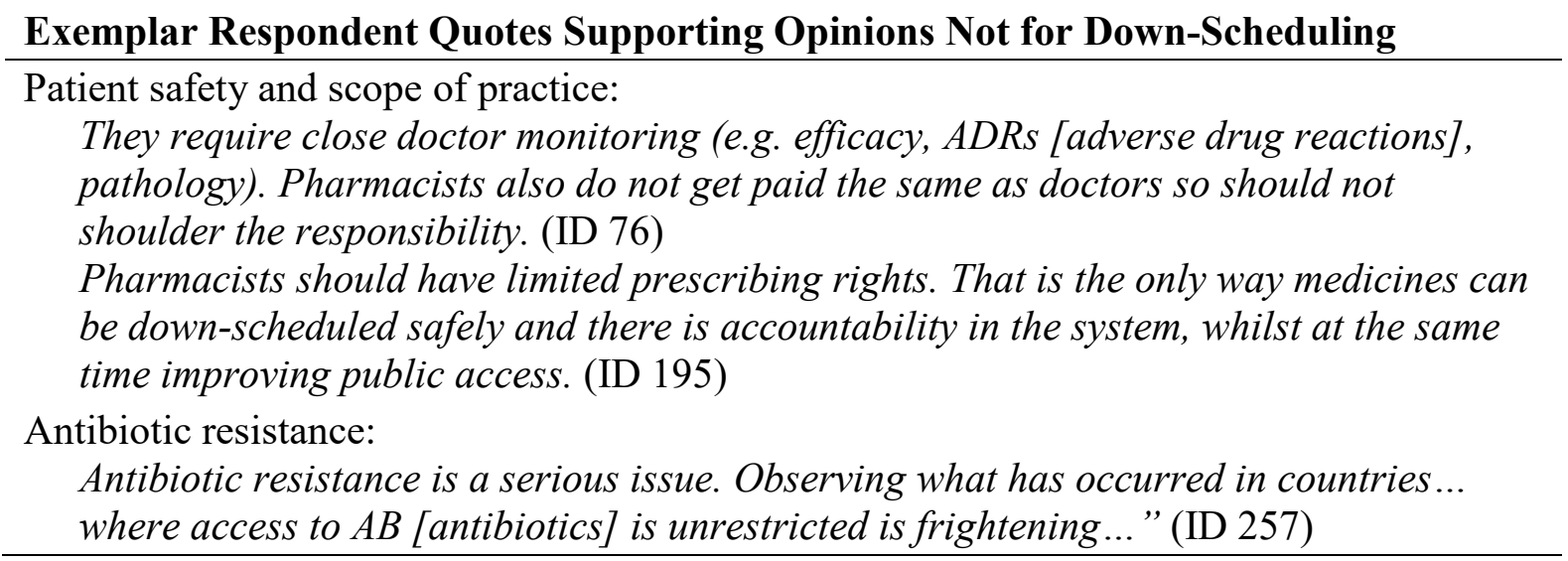

More than a third of respondents $(36.6 \%, n=86)$, identified anti-infectives as a class of medicines that should never be down-scheduled, citing concerns of overuse and the development of resistance. Other key medicines identified as not suitable for reclassification included psychotropic drugs, e.g. antidepressants and antipsychotics; cardiovascular drugs; analgesics; and groups of medicines with specific attributes. These included drugs of addiction or dependence, e.g. opioid analgesics, benzodiazepines and psychostimulants; and medicines with a narrow therapeutic index. Respondents cited concerns for patient safety, possibly unveiling that pharmacists perceived themselves as having limited skills to diagnose and capacity to conduct therapeutic drug monitoring, and limited remuneration or financial viability as justification for their opinions. When naming analgesics (particularly opioids), as medicines 
never to be considered for reclassification, pharmacists cited concerns for patient and public safety, underpinned by potential for overuse, misuse and abuse of the medicines. They were also concerned about the potential for antibiotic resistance, citing overseas experience to demonstrate potential negative outcomes of broadened access.

\section{Readiness and barriers to change}

Respondents identified the Australian pharmacy profession as ready for further downscheduling. On the readiness for change scale (where $1=$ not at all ready and $10=$ completely ready) the majority of respondents $(71.7 \%, n=137$ of 191 replies $)$ indicated between 6 and 10 , compared to $28.3 \%(\mathrm{n}=54)$ that indicated between 5 and 1 . The majority $(91.0 \%, \mathrm{n}=212)$ agreed or strongly agreed that opposition from other health professional bodies was the main barrier to further down-scheduling (mean 4.52, median 5.00). Other commonly identified barriers related to legislation or the broader health system (Table 4) including lack of access to patient's medical records, risk averseness of the medicines scheduling committee and complexity of the application process. Less commonly reported barriers included pharmacists' training, confidence, financial viability and concerns over medicines efficacy. 
Table 4: Factors Considered Barriers to Further Down-Scheduling

\begin{tabular}{lcc}
\hline Factor & Mean & Median \\
\hline Opposition from other health professional bodies & 4.52 & 5.00 \\
Lack of patient medical history & 3.79 & 4.00 \\
Risk averseness of the medicines scheduling committee & 3.78 & 4.00 \\
Complexity of reclassification application processes (i.e. red tape) & 3.77 & 4.00 \\
Concern over medicine misuse or abuse & 3.70 & 4.00 \\
Concern over inappropriate requests & 3.68 & 4.00 \\
Political conservatism & 3.59 & 4.00 \\
Concern over medicine safety & 3.48 & 4.00 \\
Lack of time for consultation & 3.44 & 4.00 \\
Lack of advocacy from peak pharmacy organisations & 3.39 & 3.00 \\
Inadequately trained support staff & 3.28 & 3.00 \\
Pharmacists are risk averse & 3.24 & 3.00 \\
Lack of training resources for pharmacists & 3.08 & 3.00 \\
Lack of pharmacist confidence in own ability & 2.88 & 3.00 \\
Current supply mechanisms are adequate* & 2.83 & 3.00 \\
Lack of financial viability & 2.82 & 3.00 \\
Concern over medicine efficacy & 2.72 & 2.00 \\
\hline
\end{tabular}

*This reflects satisfaction with the status quo

\section{Discussion}

Australian pharmacists believed their profession is ready for increased non-prescription medicines reclassification and that consumer access to selected medicines can be improved. Recommendations for less restrictive reclassification were guided by patient demand, safety and facilitating continuity of care in chronic illness. Opposition from other health professional bodies was perceived to the main barrier to reclassification.

This national cross-sectional study is the first to quantitatively and qualitatively explore Australian pharmacist perspectives on current and future reclassification. Key challenges of online surveys include more limited response rates than mail surveys ${ }^{37}$ and limitations on use of standard response rates, particularly when multiple online channels are used for recruitment. ${ }^{39}$ Researchers used varied strategies to increase response rate with promotion of the survey via a professional organisation being particularly effective. ${ }^{37}$ However, sample size, and over and under-representation of pharmacist views for selected states may limit 
generalisation of study findings. Self-reported data can be limited by respondent recall and social desirability bias and textual responses were included to capture perceptions beyond those possible with singular data collection methods.

Interestingly, pharmacist views were polarised which may highlight the contextual nature of medicines rescheduling. For example, antibiotics were frequently proposed class not to be down-scheduled, due to concerns over resistance, yet also the most frequently identified class for future reclassification. Suggestions for reclassification of antibiotics were commonly qualified in text as limited to short-course treatment of common infections, such as trimethoprim for uncomplicated UTI. This may reflect the New Zealand experience of reclassification of trimethoprim in $2012,{ }^{26}$ with reports of pharmacists acceptance of the supply model $^{25}$ and no associated reports of excessive antibiotic use or changes to usual prescribing practices. ${ }^{40}$ However, efforts to reclassify trimethoprim in the UK from Prescription Only Medicines to Pharmacist Medicines were abandoned in 2010 after concerns about resistance. ${ }^{41}$

While antibiotics were the most polarised drug class, other examples occurred of medicines being proposed for both reclassification and not for reclassification. Cardiovascular drugs were the second most reported drug class not to be down-scheduled yet lipid lowering agents and antihypertensives were also suggested as candidates for possible down-scheduling, particularly in the context of medication continuance. Some respondents included lipid lowering agents and antihypertensives in their suggestions for not being down-scheduled, but the majority of drugs identified in this category were cardiac drugs and antithrombotics, with pharmacists suggesting that these patients needed greater medical oversight and monitoring. Analgesics also appeared on the frequently reported lists for both medicines to be down-scheduled and those not to be down-scheduled. Again, the contextual nature of these apparently opposing views was that suggestions for not down-scheduling applied to analgesics with potential for abuse and misuse, e.g. opioids, whereas suggestions for potential down-scheduling applied primarily to non- 
steroidal anti-inflammatory drugs, which pharmacists considered safer and reported greater confidence in their provision.

Continuity of care was provided as a rationale for respondent recommendations of medicines to be reclassified. For example, oral contraceptives were prioritised by pharmacists and this aligns with global recommendations for non-prescription access to oral contraceptives, to prevent unintended pregnancies. ${ }^{42}$ Although recent applications to down-schedule oral contraceptives in Australia have been unsuccessful, ${ }^{43}$ selected oral contraceptives can be supplied without prescription under the PBS Continued Dispensing Initiative. ${ }^{30,44}$ In New Zealand, oral contraceptives are available without prescription, under the pharmacist-supply model applied to trimethoprim, sildenafil and oseltamivir. ${ }^{29}$

Encouragingly, the addition of Appendix M to the SUSMP may facilitate adoption of a supply model similar to that used in New Zealand ${ }^{45}$ and promote increased down-scheduling. Consultation for the proposed criteria of and medicines for inclusion in Appendix $M$ is ongoing. ${ }^{34,35,46}$ Anticipated medicines include trimethoprim for uncomplicated urinary tract infections, sildenafil for erectile dysfunction and triptans for migraine. It is expected that criteria might include additional pharmacist training and competency to be eligible to supply these medicines. Interestingly, medicines available overseas, such as sildenafil were not a priority for down-scheduling in Australia.

Patient safety was often provided as justification for why certain medicines should never be considered for down scheduling. The list of medicines or classes of medicines never to be consider for down scheduling included antibiotics and medicines that could be considered more high risk, e.g. psychotropic drugs; antineoplastic medicines and immunomodulators; and neurological drugs. Many suggested that pharmacists should not have a non-prescription supply role in any conditions that were considered complex, which might require close 
monitoring or need assessment by a healthcare team. They cautioned against rescheduling medicines with a perceived potential for abuse, misuse or diversion.

The main barrier to reclassification perceived by respondents was opposition from other health professional bodies, which was consistent with pilot interviews ${ }^{33}$ and previous Australian research that identified "patch protection" as a barrier to Australian reclassification. ${ }^{7}$ This may be influenced by a perceived loss of income as Australian medical practitioners are paid on a fee for service model. ${ }^{47}$ Respondents commonly agreed that other barriers included lack of patient medical history or risk averseness of the medicines scheduling committee. Additionally, risk averseness of pharmacists, lack of training and lack of confidence were apparent barriers, which has important implications for policy makers and training providers.

Participating pharmacists expressed overall support for policy change to enable the profession to better care for consumers with non-complex health conditions through greater access to medicines. The World Self-Medication Industry strongly supports international policy change that improves opportunities for self-care to contribute to improved health outcomes and more sustainable healthcare systems. ${ }^{48}$ Perhaps it is time to consider more creative approaches to policy change with regards to medicines reclassification. ${ }^{7}$ One creative approach to policy changes as exemplified by New Zealand, where non-sponsor stakeholders, such as pharmacy retail groups, were proactively involved in reclassification applications within models that balanced increased access with safety controls. ${ }^{8}$ It has been argued that this enabler allowed for more progressive reclassifications. ${ }^{8}$ Other identified enablers to reclassification might then be explored to facilitate further change.

To inform discussions on future policy change and goals toward medicines reclassification future research could investigate the preferred models of medicines provision and whether additional training or accreditation is sought or required, 


\section{Conclusion}

Pharmacists are clearly ready for greater reclassification yet Australia appears to have lost momentum when compared to other nations in the reclassification of medicines. The pharmacists in this study believed that their profession has the capacity to safely and effectively manage a wider range of non-prescription medicines to enhance medicines access. Pharmacists recommendations for future reclassification were context-specific and underpinned by safety and quality considerations. Given the recent creation of new regulatory structures intended to promote reclassification this study provides timely insights for Australian and international policy makers, contributing to the global conversation on non-prescription medicines access, and providing impetus for practice and policy change.

\section{Conflicts of Interest}

The authors declare no conflicts of interest.

\section{Acknowledgements}

The study was funded by The Griffith University School of Pharmacy and Pharmacology Project Grant Scheme, where all authors were employed at the time of the study. We acknowledge and thank the pharmacist participants for their time and opinions. 


\section{References}

1. Hughes CM, McElnay JC, Fleming GF. Benefits and risks of self medication. Drug safety. 2001;24(14):1027-1037.

2. Brass EP. Changing the status of drugs from prescription to over-the-counter availability. New England Journal of Medicine. 2001;345(11):810-816.

3. Noone J, Blanchette CM. The value of self-medication: summary of existing evidence. Journal of medical economics. 2018;21(2):201-211.

4. Gagnon-Arpin I. Value of Consumer Health Products: The Impact of Switching Prescription Medications to Over-the-Counter. Ottawa: The Conference Board of Canada2017.

5. Therapeutic Goods Administration. Poisons Standard (Standard for Uniform Scheduling of Medicines and Poisons) No. 23. 2019; February 2019:https://www.legislation.gov.au/Details/F2019L00032. Accessed 12 March 2019.

6. Gilbert A, Rao D, Quintrell N. A review of pharmaceutical scheduling processes in six countries and the effect on consumer access to medicines. International Journal of Pharmacy Practice. 2006;14(2):95-104.

7. Gauld N, Bryant L, Emmerton L, Kelly F, Kurosawa N, Buetow S. Why does increasing public access to medicines differ between countries? Qualitative comparison of nine countries. Journal of health services research \& policy. 2015;20(4):231-239.

8. Gauld NJ, Kelly FS, Emmerton LM, Buetow SA. Widening Consumer Access to Medicines: A Comparison of Prescription to Non-Prescription Medicine Switch in Australia and New Zealand. PLoS ONE. 2015;10(3):e0119011. 
9. National Drugs and Poisons Schedule Committee. Record of Reasons 39th Meeting 1416 October, 2003, 12.1 Levonorgestrel In. Canberra: Department of Health and Ageing; 2003:62-67, 141-147.

10. Katelaris P, Bell J. The role of the pharmacist in treating frequent heartburn: A focus on non-prescription proton pump inhibitors. Australian Pharmacist. 2017;36(4):68.

11. Alkhatib L, Parsons R, Czarniak P, Sunderland VB. An evaluation of the reclassification of ophthalmic chloramphenicol for the management of acute bacterial conjunctivitis in community pharmacies in Western Australia. International Journal of Pharmacy Practice. 2015;23(2):111-120.

12. Therapeutic Goods Administration. Scheduling delegate's final decisions: ACCS/ACMS, February 2012, $2.3 \quad$ Famciclovir pp82-106. 2012; https://www.tga.gov.au/sites/default/files/scheduling-decisions-1202-final.pdf. Accessed 30 October 2018.

13. Lenton SR, Dietze PM, Jauncey M. Australia reschedules naloxone for opioid overdose. The Medical Journal of Australia. 2016;204(4):146-147.

14. Therapeutic Goods Administration. Scheduling delegate's final decisions: ACMS\#18, July 2016, 4.1 Ulipristal. 2016; https://www.tga.gov.au/book-page/41-ulipristal. Accessed 30 October 2018.

15. Therapeutic Goods Administration. Scheduling delegate's final decisions: ACCS/ACMS, June 2013. 2013; https://www.tga.gov.au/book/part-schedulingproposals-referred-march-2013-meeting-acms\#benzo. Accessed 30 October 2018.

16. Therapeutic Goods Administration. Poisons Standard (Standard for Uniform Scheduling of Medicines and Poisons) No. 22. 2018; October 2018:https://www.legislation.gov.au/Details/F2018L01344. Accessed 30 October 2018. 
17. Medsafe New Zealand Medicines and Medical Devices Safety Authority. Committees: Database of Medicine Classifications. 2012; http://medsafe.govt.nz/profs/class/classification.asp. Accessed 30 October 2018.

18. Medsafe New Zealand Medicines and Medical Devices Safety Authority. Medicines (Class B controlled drugs) with prescribing restrictions under regulation 22 of the Misuse of Drugs Regulations 1977 - Ephedrine and Pseudoephedrine. 2011; https://medsafe.govt.nz/profs/RIss/restrict.asp\#Ephedrine. Accessed 9 June 2019.

19. Sukkar E. Risk of heart problems prompts removal of OTC diclofenac from UK pharmacy shelves. The Pharmaceutical Journal, 14 January 2015. 2015;294(7846):online URI: 20067579.

20. Gauld N, Kelly F, Emmerton L, Bryant L, Buetow S. Innovations from 'down-under': a focus on prescription to non-prescription medicine reclassification in New Zealand and Australia. SelfCare Journal. 2012;3(5):88-107.

21. Gauld NJ, Kelly FS, Kurosawa N, Bryant LJ, Emmerton LM, Buetow SA. Widening Consumer Access to Medicines through Switching Medicines to Non-Prescription: A Six Country Comparison. PloS one. 2014;9(9):e107726.

22. Sukkar E. Drug Switching Q\&A: OTC medicines give power to the patient. The Pharmaceutical Journal, 15 November 2014. 2014;293(7835):online URI: 20067092.

23. ANZTPA. The Treaty: Agreement between the Government of Australia and the Government of New Zealand for the establishment of a joint scheme for the regulation of therapeutic products. 2003.

24. ANZTPA. Transition to the Australia New Zealand Therapeutic Products Agency (ANZTPA). 2014; http://www.anztpa.org/index.htm. Accessed 30 October 2018. 
25. Braund R, Henderson E, McNab E, Sarten R, Wallace E, Gauld N. Pharmacist-only trimethoprim: pharmacist satisfaction on their training and the impact on their practice. International journal of clinical pharmacy. 2016;38(6):1357-1361.

26. Gauld NJ. Improving access to urinary tract infection treatment: The reclassification of trimethoprim. SelfCare Journal. 2012;3(6):115-120.

27. Ahmed S, Rutter P. UK community pharmacists experiences on over-the-counter tamsulosin. SelfCare. 2011;2(6):152-159.

28. Karimkhani C, Dellavalle R. Why we need more over-the-counter products to treat acne. The Pharmaceutical Journal. 2016(7 Oct).

29. Gauld N. Pharmacists to supply oral contraceptives in NZ. The Pharmaceutical Journal. 2017(9 March).

30. Australian Government Department of Human Services. Education guide - Continued $\begin{array}{lllll}\text { Dispensing } \quad \text { Initiative } & - & \text { PBS } & \text { requirements. }\end{array}$ https://www.humanservices.gov.au/health-professionals/enablers/education-guidecontinued-dispensing-initiative-pbs-requirements. Accessed 30 October 2018.

31. NHS Scotland. NHS Inform: Pharmacy services. 2019; https://www.nhsinform.scot/care-support-and-rights/nhsservices/pharmacy/pharmacy-services. Accessed 7 January 2019.

32. Gauld N, Sullivan T. Double-Dosing and Other Dangers with Non-Prescription Medicines: Pharmacists' Views and Experiences. Pharmacy. 2018;6(3):59.

33. Mey A, King M, Kelly F, et al. Australian pharmacy perspectives on increasing access to medicines through reclassification. Journal of Health Services Research \& Policy. 2018;0(0):1-10.

34. Therapeutic Goods Administration. Consultation: Proposed criteria for Appendix M of the Poisons Standard to support rescheduling of substances from Schedule 4 
(Prescription only) to Schedule 3 (Pharmacist only). 2019; Version 1.0, February 2019:https://www.tga.gov.au/sites/default/files/consultation-proposed-criteriaappendix-m-poisons-standard-support-rescheduling-substances-schedule-4prescription-only-schedule-3-pharmacist-only.pdf. Accessed 13 March 2019.

35. Hope DL, Mey A, Kelly FS, King MA. Sorting out the Schedule. Australian Journal of Pharmacy. 2019(April):22-26.

36. Lotfipour S, Cisneros V, Chakravarthy B, et al. Assessment of readiness to change and relationship to AUDIT score in a trauma population utilizing computerized alcohol screening and brief intervention. Substance abuse. 2012;33(4):378-386.

37. Fan W, Yan Z. Factors affecting response rates of the web survey: A systematic review. Computers in Human Behavior. 2010;26(2):132-139.

38. WHO Collaborating Centre for Drugs Statistics Methodology. ATC Structure and Principles. 2011; https://www.whocc.no/atc/structure and principles/. Accessed 30 October 2018.

39. Eysenbach G. Improving the quality of Web surveys: the Checklist for Reporting Results of Internet E-Surveys (CHERRIES). Journal of medical Internet research. 2004;6(3):e34.

40. Gauld NJ, Zeng IS, Ikram RB, Thomas MG, Buetow SA. Antibiotic treatment of women with uncomplicated cystitis before and after allowing pharmacist-supply of trimethoprim. International Journal of Clinical Pharmacy. 2017;39(1):165-172.

41. The Pharmaceutical Journal. Trimethoprim and nitrofurantoin POM-to-P switches abandoned. Vol. 284, p417 2010; https://www.pharmaceutical-journal.com/news-andanalysis/news/trimethoprim-and-nitrofurantoin-pom-to-pswitchesabandoned/11008849.article. Accessed 10 December 2018. 
42. McIntosh J, Rafie S, Wasik M, et al. Changing oral contraceptives from prescription to over-the-counter status: an opinion statement of the Women's Health Practice and Research Network of the American College of Clinical Pharmacy. Pharmacotherapy: The Journal of Human Pharmacology and Drug Therapy. 2011;31(4):424-437.

43. Therapeutic Goods Administration. Scheduling delegate's interim decisions and invitation for further comment: ACMS, June 2015 1.1 Oral contraceptive pill. 2015; https://www.tga.gov.au/book/part-interim-decisions-matters-referred-expert-advisorycommittee-acms14-11-13. Accessed 30 October 2018.

44. Hope DL, Dickfos ST, Ellerby RE, King MA. Borderline Health: Potential Risks to Patients and Practitioners of Jurisdictional Variation in Australian Medicines Legislation. Journal of Pharmacy Practice and Research. 2016;46(3):201-208.

45. Medsafe New Zealand Medicines and Medical Devices Safety Authority. Medicines: Pharmacist-Only Medicines. 2013; http://www.medsafe.govt.nz/Consumers/PharmOnly.asp. Accessed 12 March 2019.

46. Hope DL, Mey A, Kelly FS, King MA. Appendix M: What Do Pharmacists Want? Australian Journal of Pharmacy 2019; https://ajp.com.au/wpcontent/uploads/2019/04/Appendix-M-Paper-FINAL.pdf.

47. Australian Government Department of Health. MBS Online - Medicare Benefits Schedule. 2019; http://www.mbsonline.gov.au/internet/mbsonline/publishing.nsf/Content/Downloads201907. Accessed 9 June 2019.

48. World Self-Medication Industry. WSMI: Who We Are, Vision, Mission and Work Focus. 2019; http://www.wsmi.org/about-wsmi/who-we-are-vision-mission-andwork-focus/. Accessed 7 January 2019. 